\title{
Gold nanorods grown from HgTe nanoparticles directly on various surfaces
}

\author{
S. I. Shopova, C. W. Blackledge, and A. T. Rosenberger ${ }^{\text {a) }}$ \\ Department of Physics, Oklahoma State University, Stillwater, Oklahoma 74078-3072 \\ N. F. Materer \\ Department of Chemistry, Oklahoma State University, Stillwater, Oklahoma 74078-3071
}

(Received 23 November 2005; accepted 25 May 2006; published online 14 July 2006)

\begin{abstract}
Gold nanorods (NRs) are nucleated by HgTe semiconductor nanoparticles. Growth of $\sim 200$ $\times 50 \mathrm{~nm}^{2}$ NRs directly on various surfaces is achieved by using an intermediary polyelectrolyte layer. X-ray photoelectron spectroscopy confirms the deposition of gold. An increase in the intensity of the Au NR plasmon resonance is observed with optical extinction spectroscopy. This seeding technique, amenable to many different surfaces, suggests a simple synthetic route to composite materials with interesting electronic and optical properties. (C) 2006 American Institute of Physics.
\end{abstract}

[DOI: 10.1063/1.2221889]

We demonstrate a general method to nucleate Au nanorods (NRs) using thioglycolic acid (TGA) capped HgTe nanoparticles (NPs) adsorbed to a polydimethyldiallyammonium (PDDA) chloride layer on a variety of surfaces including dielectric microspheres. In the last few years, several reports have discussed Au NR synthesis on surfaces. ${ }^{1-3}$ Growth on surfaces has brought attention to the growth mechanism, and surface immobilization is required for many foreseeable optical applications. The longitudinal plasmon resonance of $\mathrm{Au}$ NRs is of particular interest because it is tunable, sensitive to its environment, and enhances near-field optical effects. Heterogeneous nucleation of Au NRs using nonmetallic seeds such as semiconductor quantum dots suggests the possibility of synthesizing composite nanomaterials with interesting optical and electronic properties. Mercury telluride nanoparticles have size tunable electronic states in the same spectral region as the longitudinal mode of the $\mathrm{Au}$ NR plasmon.

Transmission electron microscopy (TEM), scanning electron microscopy (SEM), atomic force microscopy (AFM), visible and near infrared extinction spectroscopies, energy dispersive atomic $\mathrm{x}$-ray spectroscopy (EDX), and $\mathrm{x}$-ray photoelectron spectroscopy (XPS) are used to characterize the samples. TEM is performed with a JEOL (JEM 100 CX II) at $100 \mathrm{kV}$ and SEM with a JEOL (JXM 6400) at $30 \mathrm{kV}$. AFM results are from a Digital Instruments Nanoscope IIIa. The extinction spectroscopy experiments are carried out using a Varian Cary 5. EDX measurements are from an Evex Analytical System. Finally, XPS measurements are performed with a Mg anode and a PHI double-pass cylindrical mirror analyzer with a pass energy of $50 \mathrm{eV}$. Hydrogen tetrachloroauric acid, cetyl trimethyl ammonium bromide (CTAB), ascorbic acid, polydimethyldiallyammonium (PDDA) chloride, and silver nitrate were from SigmaAldrich. Aqueous TGA capped HgTe NPs were synthesized as described by Rogach et al. ${ }^{4} 18 \mathrm{M} \Omega$ water (Labconco Water Pro Plus) was used.

The procedure for growing $\mathrm{Au}$ NRs from HgTe NPs bound to surfaces is depicted in Figs. 1(a)-1(c). First, the surface is dipped in an aqueous solution of $0.5 \%$ PDDA for $\sim 30$ min [Fig. 1(a)]. After washing with de-ionized water,

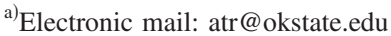

the substrate is dipped in an aqueous solution of TGA capped HgTe NPs for $\sim 5-10 \mathrm{~s}$ [Fig. 1(b)]. ${ }^{5,6}$ The concentration of seeds on the surface is kept low, approximately 20-200 NPs $/ \mu \mathrm{m}^{2}$, to enable the growth of well separated $\mathrm{Au}$ rods. The negatively charged carboxylate group of the HgTe NP capping agent is electrostatically bound to the quaternary amines of PDDA. ${ }^{5}$ After washing with de-ionized water, the substrates are immersed in a gold growth solution of $1.3 \times 10^{-4} \mathrm{M} \mathrm{HAuCl} 4,8.0 \times 10^{-3} \mathrm{M}$ CTAB, and 1.9 $\times 10^{-4} \mathrm{M}$ ascorbic acid. The ascorbic acid reduces $\mathrm{AuCl}_{4}^{-}$to $\mathrm{AuCl}_{2}^{-}$and other $\mathrm{Au}^{+}$species before the substrate is dipped into the solution for up to $45 \mathrm{~min}$. Further reduction to $\mathrm{Au}^{0}$ is induced by the HgTe NP seeds. After $45 \mathrm{~min}$ the initially clear substrates acquire a dark purple to blue color while the solution remains colorless. The sample is washed with deionized water again to remove the excess CTAB on the surface. Furthermore, when microsphere samples are sonicated for up to $3 \mathrm{~min}$ in water to remove adsorbed CTAB and improve their optical quality, their color does not significantly change. Figure 1(c) tentatively depicts the seed remaining at one end of the NR as it induces rod growth. The

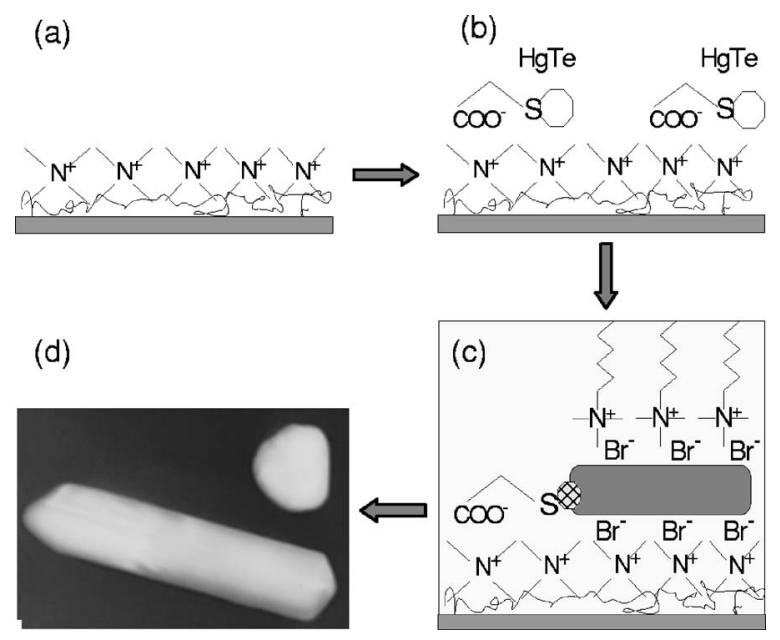

FIG. 1. HgTe NP seeded Au NR growth on surfaces. (a) The substrate is coated with a monolayer of PDDA, washed with de-ionized water, and dried. (b) A submonolayer of HgTe NPs, capped with TGA, is formed on top of the PDDA layer. (c) The sample is immersed in Au growth solution, where one-dimensional growth is assisted by CTAB. (d) TEM image of resulting shapes. The rod is $330 \mathrm{~nm}$ long and has an aspect ratio of 5 . 

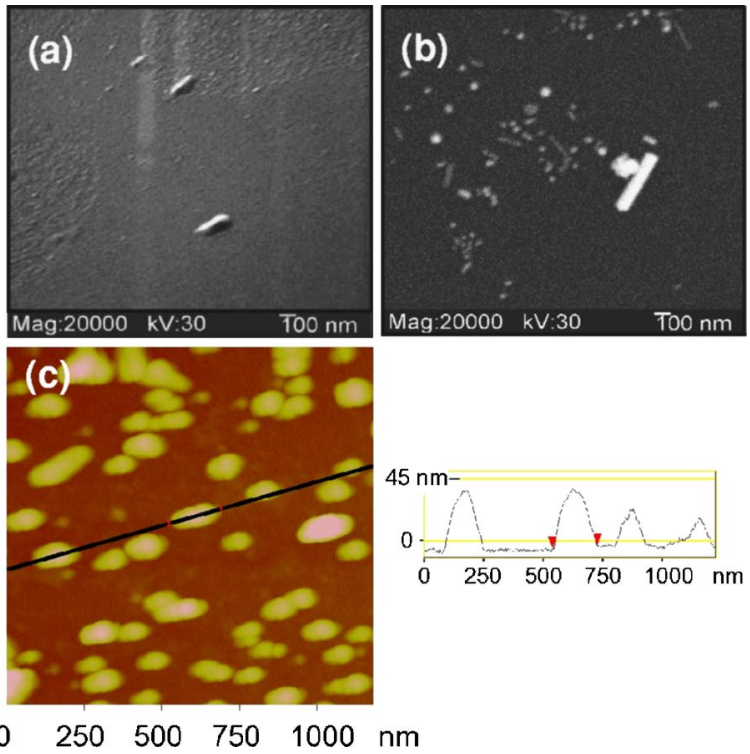

FIG. 2. (Color online) (a) SEM image of the Au NRs grown on the surface of a fused silica microsphere of diameter $450 \mu \mathrm{m}$. (b) SEM of rods grown on a mica surface. (c) AFM image of rods grown on a glass substrate, with a trace of the height profile along the dark line.

seed and rod are not to scale in Fig. 1(c); the diameter of the HgTe seed is actually about one-tenth the width of the rod. A TEM image of two resulting particles, a NR and a NP, is given in Fig. 1(d). The typical NRs observed have sharp tips. This is a desirable shape for greater electric field enhancement.

As control experiments, clean glass substrates, PDDA coated glass slides, and PDDA coated silica microspheres of $\sim 500 \mu \mathrm{m}$ diameter are exposed to the gold growth solution. No change of color is observed on the sample or in the solution during $45 \mathrm{~min}$ of exposure. Further examination of the initially PDDA coated samples with SEM and absorption spectroscopy does not show growth of gold particles on the surface. Also no color change is observed in $45 \mathrm{~min}$ when PDDA or the sodium salt of 2-thiobenzoic acid (sodium mercaptobenzoate) is added separately or together with the gold growth solution. So, in the absence of HgTe seeds, bare substrates, PDDA, and water soluble thiol-containing species such as TGA do not induce nucleation of nanoparticulate $\mathrm{Au}^{0}$.

Figure 2(a) is a SEM image of a sample made with a low density of HgTe NPs bound to the surface of a silica microsphere, and EDX confirms the presence of $\mathrm{Au}, \mathrm{Hg}$, and $\mathrm{Te}$. Figure 2(b) shows SEM results of a sample grown on mica, and Fig. 2(c) shows AFM results on glass. The particle shapes and sizes are similar to reports of Au NRs nucleated on surfaces using Au NPs. ${ }^{3}$ Using TEM and SEM, measured NR lengths are $235 \pm 90 \mathrm{~nm}$, widths are $50 \pm 20 \mathrm{~nm}$, and aspect ratios (length/width) are $4.7 \pm 2.3$. AFM measurements yield lengths of $153 \pm 43 \mathrm{~nm}$, widths of $70 \pm 11 \mathrm{~nm}$, and heights of $36 \pm 6 \mathrm{~nm}$, with aspect ratios (length/height) of $4.3 \pm 1.3$, as seen in the trace from Fig. 2(c).

Figure 3 shows the evolution of the visible and near infrared extinction spectra of samples grown on glass substrates. The samples are prepared by removing the substrates from the Au growth solution at various times, rinsing with water, and drying in air. The spectrum of the unseeded PDDA sample treated with Au growth solution confirms that $\mathrm{Au}$ NR nucleation does not occur without HgTe NPs. An Downloaded 18 Jul 2006 to 139.78 .127 .123 . Redistribution subject

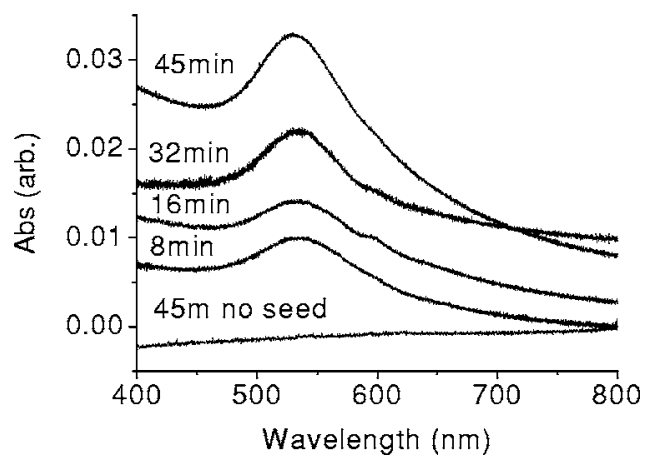

FIG. 3. Extinction spectra of Au deposited on four different glass surfaces using HgTe NPs as nucleation seeds.

increase in the intensity of the plasmon bands of Au NRs and NPs is observed as the reaction proceeds. The feature at $540 \mathrm{~nm}$ is from the transverse plasmon mode of Au NRs and the plasmon of isotropic Au NPs. The extinction at longer wavelengths is from the longitudinal modes of rods and multipole coupling between particles. A pronounced peak from a longitudinal mode is not observed in the spectral range up to $800 \mathrm{~nm}$. However, the spectra of Fig. 3 are comparable to $\mathrm{Au}$ NRs seeded directly on surfaces using Au NPs. ${ }^{2}$ As the growth progresses, Au particles form a dense surface coverage and the longitudinal plasmon is broadened and redshifted by multipole coupling between particles. Variability in the particle density and fractional yield of NRs/NPs can explain the different background intensities in the various extinction spectra.

Additional evidence that $\mathrm{Au}$ NRs are nucleated by $\mathrm{HgTe}$ NPs is provided by XPS. Figure 4(a) shows the increase in the Au $4 f$ spin-orbit doublet at binding energies of 83 and $87 \mathrm{eV}$ as a function of reaction time for a sample prepared on a glass substrate; the signal from the $\mathrm{Hg} 4 f$ spin-orbit doublet at 104 and $100 \mathrm{eV}$ is unfortunately overlapped by a large $\mathrm{Si} 2 p$ signal at $103 \mathrm{eV}$ due to the glass substrate. In Fig. 4(b), the intensity of the Te $3 d_{5 / 2}$ peak (which overlaps the less intense $\mathrm{Hg} 4 p_{3 / 2}$ peak) is given at a larger scale, as evident from the noise. The ratio of the Te to $\mathrm{Si}$ peaks remains constant, and the ratio of $\mathrm{Au}$ to $\mathrm{Si}$ peaks grows with time. The relatively low $\mathrm{Au} / \mathrm{Te}$ ratio (below 10) suggests that not all the seeds are contributing to the nucleation of gold. However, the XPS results indicate growth of Au, while there is very little change in the signals from the substrate (Si) and the HgTe NPs.

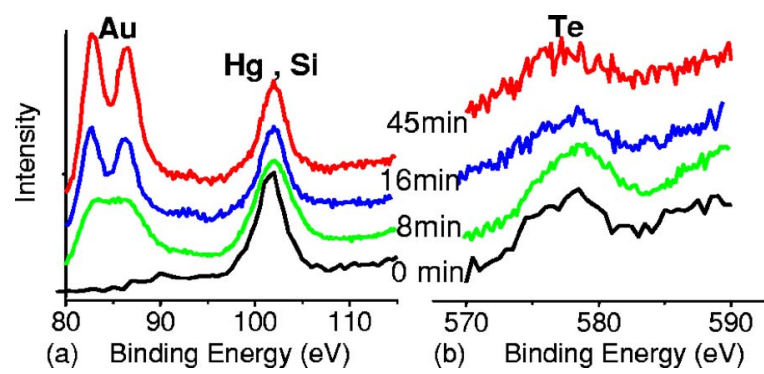

FIG. 4. (Color online) XPS of samples of HgTe NPs adsorbed on glass and exposed to Au growth solution for $0,8,16$, and $45 \mathrm{~min}$. Spectra are normalized and offset for clarity. (a) The Au $4 f$ spin-orbit doublet at 83 and $87 \mathrm{eV}$, and the overlapping $\mathrm{Hg} 4 f$ (from the nucleation particles) and $\mathrm{Si} 2 p$ (from the substrate) peaks at $102 \mathrm{eV}$. (b) The Te $3 d_{5 / 2}$ peak (from the nucleation particles) at $580-575 \mathrm{eV}$.

AIP license or copyright, see http://apl.aip.org/apl/copyright.jsp 
The Au NR nucleation and growth mechanism is still a subject under intense investigation. ${ }^{7,8}$ Typically, small $(\sim 3 \mathrm{~nm}$ diameter) Au NPs are used as seeds. Many reports suggest that before anisotropic growth begins, the seeds grow to form multifaceted $\mathrm{Au}$ particles of approximately $20 \mathrm{~nm}$ in diameter ${ }^{9,10}$ and that the transition to rod growth is aided by the presence of crystal defects such as twinning planes. ${ }^{10,11}$ An arbitrary nucleation seed such as $\mathrm{HgTe}$ will result in more elastic strain and crystal defects in the first few layers of $\mathrm{Au}$ atoms than a $\mathrm{Au}$ seed. $\mathrm{HgTe}$ and Au have lattice constants of 0.65 and $0.41 \mathrm{~nm}$, respectively. The lattice mismatch of $\mathrm{HgTe}$ and $\mathrm{Au}$ may explain the greater ratio of NRs to isotropic particles when these results are compared to other work. Based on SEM and TEM images of different substrates, $30 \% \pm 8 \%$ of the particles using $\mathrm{HgTe}$ seeds are rod shaped (aspect ratio $>2$ ), in comparison to $15 \%$ or less in other work that uses Au seeds. ${ }^{2,3}$ Lattice mismatch between the seed and gold also thermodynamically favors the seed to be located on an outer surface of the Au NR to reduce the area of contact and minimize strain. Furthermore the strained boundary is a site of favorable addition of Au during growth.

In the evolving model of colloidal Au NR growth, CTAB binds selectively to $\mathrm{Au}$ faces, especially the otherwise unstable (110) surface. ${ }^{7,12}$ The surfactant CTAB forms an interdigitated bilayer that retards diffusion of solvated Au species to the bound faces of the NR. Bromide ions and water bridge the NR surface to the quaternary amines of $\mathrm{CTA}^{+} .{ }^{12}$ The intermediate PDDA layer used to immobilize the HgTe NPs also contains positively charged quaternary amines. Unlike previous substrates used in surface bound nucleation of $\mathrm{Au}$ NRs, the quaternary amines of PDDA compete with $\mathrm{CTA}^{+}$ for the $\mathrm{Br}^{-}$ions around the Au surface. As a result, strong binding of Au NRs to PDDA stabilizes the final pattern of the ensemble; the surfaces maintain their color when they are sonicated after the growth of Au. This is a desirable result for applications that require an enduring NR configuration, such as on microsphere resonators.
In conclusion, the synthesis of Au NRs using HgTe NPs as nucleation seeds is demonstrated. A versatile quaternary amine-containing polymer, PDDA, is used to attach the seeds and immobilize the resulting NRs to various surfaces. Samples are characterized with SEM, EDX, TEM, AFM, XPS, and extinction spectroscopy. Rods with aspect ratios in the range of 2-7 are obtained. Heterogeneous nucleation of $\mathrm{Au}$ NRs with semiconductor NPs will help elucidate the mechanism of surfactant-aided and seed-induced NR synthesis, and suggests a straightforward bench-top procedure to synthesize nanoscale composites.

This work was supported by the National Science Foundation under Awards Nos. ECS-0115442, ECS-0329924, and EPS-0132534. The authors thank Dr. Nicholas A. Kotov for supplying the HgTe NPs, and Dr. Bret N. Flanders, Dr. Joel J. Martin, and Dr. James P. Wicksted for laboratory space and loan of some equipment.

${ }^{1}$ H. Liao and J. Hafner, J. Phys. Chem. B 108, 19276 (2004).

${ }^{2}$ Z. Wei, A. Mieszawska, and F. Zamborini, Langmuir 20, 4322 (2004).

${ }^{3}$ N. Taub, O. Krichevski, and G. Markovich, J. Phys. Chem. B 107, 11579 (2003).

${ }^{4}$ A. Rogach, S. Kershaw, M. Burt, M. Harrison, A. Kornowski, A. Eychmuller, and H. Weller, Adv. Mater. (Weinheim, Ger.) 11, 552 (1999).

${ }^{5}$ A. L. Rogach, D. S. Koktysh, M. Harrison, and N. A. Kotov, Chem. Mater. 12, 1526 (2000).

${ }^{6}$ S. I. Shopova, G. Farca, A. T. Rosenberger, W. M. S. Wickramanayake, and N. A. Kotov, Appl. Phys. Lett. 85, 6101 (2004).

${ }^{7}$ C. J. Murphy, T. K. Sau, A. M. Gole, C. J. Orendorff, J. Gao, L. Gou, S. E. Hunyadi, and T. Li, J. Phys. Chem. B 109, 13857 (2005).

${ }^{8}$ J. Perez-Juste, L. Liz-Marzan, S. Carnie, D. Chan, and P. Mulvaney, Adv. Funct. Mater. 14, 571 (2004); M. Liu and P. Guyot-Sionnest, J. Phys. Chem. B 109, 22192 (2005).

${ }^{9}$ C. Johnson, E. Dujardin, S. Davis, C. Murphy, and S. Mann, J. Mater. Chem. 12, 1765 (2002); S. Zhang, Z. Jiang, Z. Xie, X. Xu, R. Huang, and L. Zheng, J. Phys. Chem. B 109, 9416 (2005).

${ }^{10}$ P. Gai and M. Harmer, Nano Lett. 2, 771 (2002).

${ }^{11}$ C. Lofton and W. Sigmund, Adv. Funct. Mater. 15, 1197 (2005).

${ }^{12}$ B. Nikoobakht and M. El-Sayed, Langmuir 17, 6368 (2001). 